\title{
Delayed treatment of ruptured brain AVMs: is it ok to wait?
}

\author{
Jeffrey S. Beecher, DO, Kristopher Lyon, MD, Vin Shen Ban, MBBChir, MRCS, MSc, \\ Awais Vance, MD, Cameron M. McDougall, MD, Louis A. Whitworth, MD, Jonathan A. White, MD, \\ Duke Samson, MD, H. Hunt Batjer, MD, and Babu G. Welch, MD
}

Department of Neurological Surgery, UT Southwestern Medical Center, Dallas, Texas

\begin{abstract}
OBJECTIVE Despite a hemorrhagic presentation, many patients with arteriovenous malformations (AVMs) do not require emergency resection. The timing of definitive management is not standardized in the cerebrovascular community. This study was designed to evaluate the safety of delaying AVM treatment in clinically stable patients with a new hemorrhagic presentation. The authors examined the rate of rehemorrhage or neurological decline in a cohort of patients with ruptured brain AVMs during a period of time posthemorrhage.
\end{abstract}

METHODS Patients presenting to the authors' institution from January 2000 to December 2015 with ruptured brain AVMs treated at least 4 weeks posthemorrhage were included in this analysis. Exclusion criteria were ruptured AVMs that required emergency surgery involving resection of the AVM, prior treatment of AVM at another institution, or treatment of lesions within 4 weeks for other reasons (subacute surgery). The primary outcome measure was time from initial hemorrhage to treatment failure (defined as rehemorrhage or neurological decline as a direct result of the AVM). Patientdays were calculated from the day of initial rupture until the day AVM treatment was initiated or treatment failed.

RESULTS Of 102 ruptured AVMs in 102 patients meeting inclusion criteria, $7(6.9 \%)$ failed the treatment paradigm. Six patients (5.8\%) had a new hemorrhage within a median of 248 days (interquartile range 33-1364 days). The total "at risk" period was 18,740 patient-days, yielding a rehemorrhage rate of $11.5 \%$ per patient-year, or $0.96 \%$ per patient-month. Twelve (11.8\%) of 102 patients were found to have an associated aneurysm. In this group there was a single (8.3\%) new hemorrhage during a total at-risk period of 263 patient-days until the aneurysm was secured, yielding a rehemorrhage risk of $11.4 \%$ per patient-month.

CONCLUSIONS It is the authors' practice to rehabilitate patients after brain AVM rupture with a plan for elective treatment of the AVM. The present data are useful in that the findings quantify the risk of the authors' treatment strategy. These findings indicate that delaying intervention for at least 4 weeks after the initial hemorrhage subjects the patient to a low $(<1 \%)$ risk of rehemorrhage. The authors modified the treatment paradigm when a high-risk feature, such as an associated intracranial aneurysm, was identified.

https://thejns.org/doi/abs/10.3171/2017.1.JNS16745

KEY WORDS arteriovenous malformation; delayed surgery; rehemorrhage risk; vascular disorders

$\mathrm{A}$ RTERIOVENOUS malformations (AVMs) are lesions characterized by the presence of a nidus containing an abnormal array of tortuous vascular channels between feeding arteries and draining veins without an intervening capillary network. ${ }^{6}$ These congenital lesions are relatively rare, with an approximate incidence of 1 in 100,000 people. ${ }^{7,826}$ AVMs most commonly present with intracranial hemorrhage. The mortality rate related to these hemorrhages is approximately $10 \%-30 \% .^{1,3}$ The annual risk of rehemorrhage ranges from $3 \%$ to $15 \%$. Most physicians suggest that definitive treatment be considered once a patient with an AVM presents with a hemorrhage. ${ }^{5,9,10,12,25,29}$

Emergency surgery should be considered for a patient when a rapidly declining neurological status is attributed to a ruptured AVM. ${ }^{13,16,21,22,27}$ Clinical judgment should dictate whether the acute surgery requires resection of the AVM or only decompression to stabilize the patient's

ABBREVIATIONS AVM = arteriovenous malformation; $G O S$ = Glasgow Outcome Scale; IA = intracranial aneurysm; IQR = interquartile range.

SUBMITTED July 13, 2016. ACCEPTED January 26, 2017.

INCLUDE WHEN CITING Published online July 7, 2017; DOI: 10.3171/2017.1.JNS16745. 
condition. The critical elements involved in this surgical decision are the clinical condition of the patient, the ability to better characterize the AVM, and the facility where the patient presents. There are some reports in the literature that patients with ruptured, low-grade AVMs have a reduced hospital stay and have more rapid rehabilitative course when AVM resection is performed in the first 6 days after rupture. ${ }^{19}$ To the contrary, delayed, elective treatment may avoid the risk of exacerbating the deficit(s) that brought the patient to medical attention. ${ }^{17,18,28}$

Similar to the historical management of cerebral aneurysms, surgical management of AVMs followed the principle of performing surgery once the patient's brain had been able to recover from the initial injury. ${ }^{20,24}$ Classic studies by Kassell and Torner ${ }^{14}$ and Inagawa et al. ${ }^{11}$ confirmed a higher aneurysm rehemorrhage rate in the 1st week. As a result, contemporary aneurysm management occurs within 48-72 hours. ${ }^{4,15}$ The rerupture risk of AVMs ranges from $6 \%$ to $15.8 \%$ in the 1st year and from $2 \%$ to $7.9 \%$ each subsequent year, making an elective treatment approach more feasible. , $7,9,26,29^{2}$

Intracranial aneurysms (IAs) occur in $7 \%-20 \%$ of patients with AVMs. ${ }^{718}$ We have previously described our classification system of aneurysms associated with AVMs.? They are classified as Type I, IIa, IIb, and III based on their relative proximity to the nidus of the AVM (Fig. 1). Type I aneurysms are remote from the AVM, Type II are located on a feeding vessel and considered flow related (Type IIa, proximal; Type IIb, distal), and Type III an- eurysms are intranidal. In this study, we maintained this classification and reviewed the subset of patients with aneurysms relevant to the AVM.

The American Heart Association guidelines recommend that surgery for ruptured AVMs should be elective. There is an absence of literature detailing the safety of this strategy, and there is no recommended timeline. ${ }^{18}$ Our institution has employed the treatment paradigm described below since the conception of our database in the 1990s, and we recognize that the cerebrovascular community manages ruptured AVMs largely in a similar manner. However, the literature lacks a review and evaluation of risk incurred by the patient during the period prior to treatment. Also, the guidelines using "elective" as the descriptor of when surgery should be performed are vague. It is our opinion that there should be a consensus in the literature regarding the timing of AVM treatment after initial rupture that aims to minimize rerupture risk and risks associated with early surgery. The aim of this study was to better understand the risk of intentionally delayed treatment of a ruptured AVM by quantifying the risk assumed by the patient during the time interval from initial hemorrhage to treatment initiation.

\section{Methods}

A retrospective review of patients with brain AVMs admitted to our institution from January 2000 to December 2015 was performed. The study was approved by our
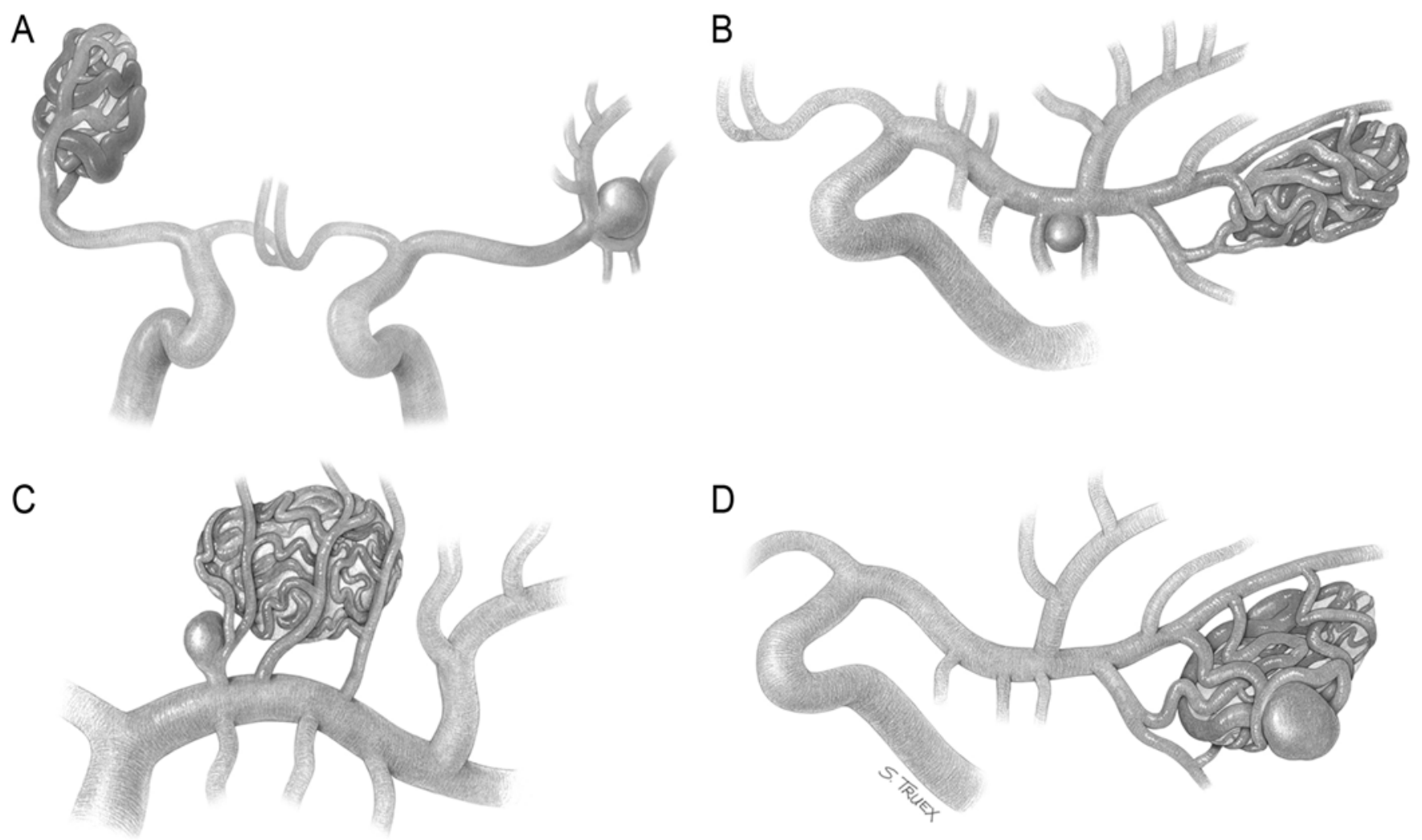

FIG. 1. Types of aneurysms associated with AVMs classified by location with respect to the AVM: Type I unrelated (A), Type lla proximal flow-related (B), Type Ilb distal flow-related (C), and Type III intranidal (D) lesions. Artist: Suzanne Truex. Copyright UT Southwestern, Department of Neurological Surgery. Published with permission. 
institutional review board. The inclusion criteria were patients of all ages presenting with any ruptured brain AVM treated in a delayed fashion. For the purposes of the study, a delayed fashion was considered a delay of at least 4 weeks after the initial hemorrhage. During this time the patients were maintained in an intensive care environment until considered stable for transition to rehabilitation or to a caregiver at home. Patients were excluded if they presented with a ruptured brain AVM that was treated with emergency surgery involving resection of the AVM, if they had a history AVM management prior to presentation, if they had rupture from a Type I or IIa aneurysm, or if they were electively treated less than 4 weeks after the hemorrhage date (subacute treatment). Patients were not excluded based upon the treatment modality they were assigned to as long as the treatment occurred 4 weeks after the patient's index hemorrhage (Fig. 2). A patient was included if, during the delay, they suffered rehemorrhage or decompensation secondary to the AVM. This was considered a failure of the treatment paradigm.

A subgroup of patients was identified and categorized separately for review. These patients harbored an IA associated with the brain AVM. Patients were included when a distal flow-related or intranidal aneurysm (Type IIb or III) was identified. This shifted the treatment paradigm to acute or subacute treatment of the aneurysm followed by AVM management in our usual delayed fashion.
Statistical analyses were performed in SPSS v20 (IBM Corporation). A stepwise logistic regression analysis was performed to identify predictors of rehemorrhage. Significance was set at 0.05 .

\section{Results}

Five hundred eighty-one patients presented to our institution from 2000 to 2015 with an AVM. One hundred sixty-three patients $(28.1 \%)$ had a hemorrhagic presentation, and 102 met the inclusion criteria for the primary analysis. The remaining 61 patients were excluded due to emergency AVM treatment $(n=6)$, early elective treatment $(n=14)$, prior treatment at an outside institution $(n=9)$, unknown hemorrhage date $(\mathrm{n}=15)$, unknown rehemorrhage date $(\mathrm{n}$ = 1), unknown AVM treatment date or lost to follow-up (n $=15)$, and unknown treatment details $(\mathrm{n}=1)$.

The characteristics of the 102 included patients are summarized in Table 1. The median and mean delays to treatment were 3.5 and 6.1 months, respectively. Eightytwo percent of patients were treated within 6 months. Reasons for delay included delayed referral from another institution/provider, need for additional diagnostic imaging due to persistent hematoma, patient preference, pregnancy, and lost to follow-up. For delay in treatment greater than 6 months ( $\mathrm{n}=18$ patients), the most common reason was delayed referral $(\mathrm{n}=11)$, and this was followed by patient

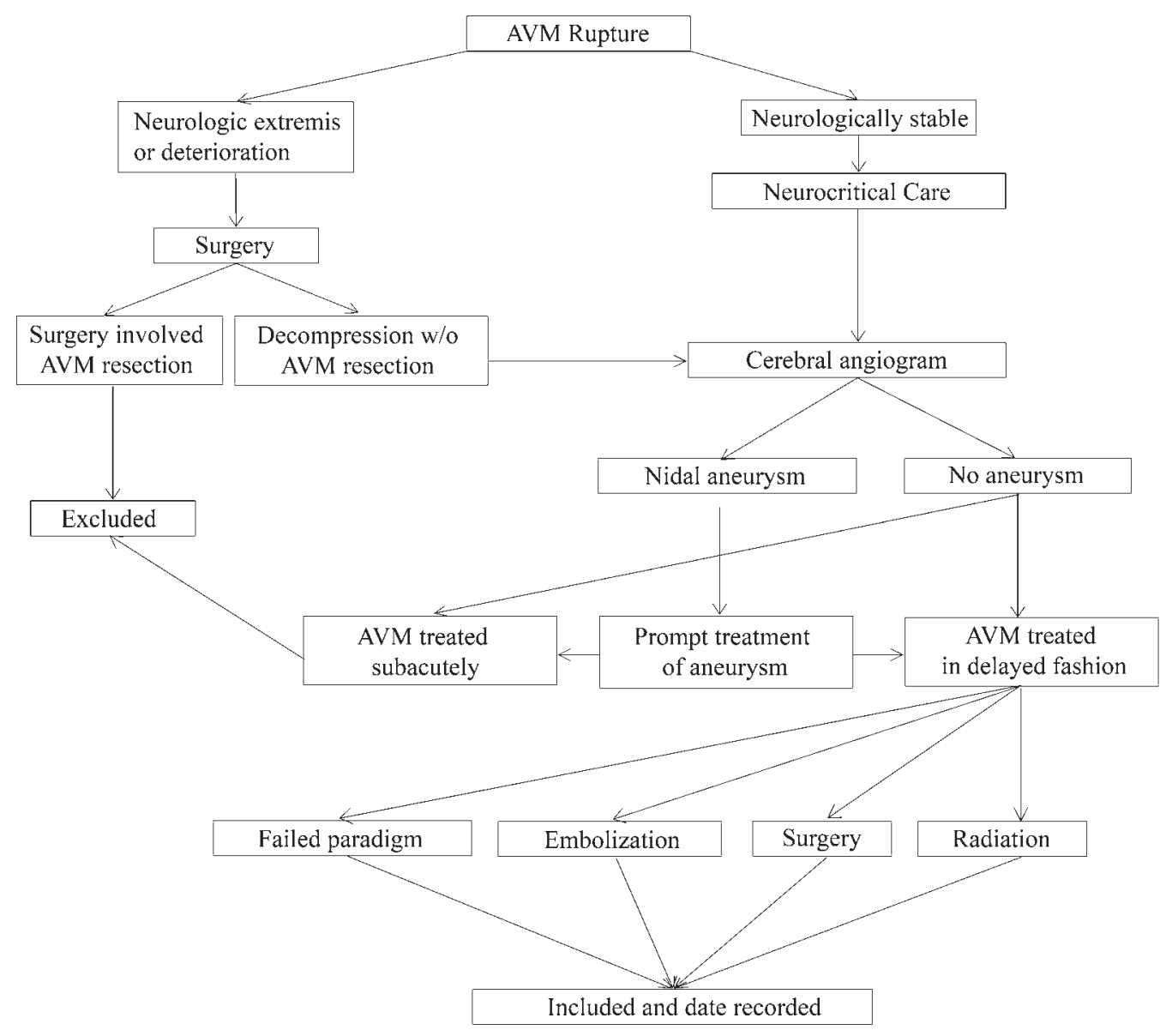

FIG. 2. Treatment paradigm and patient inclusion process. 
TABLE 1. Descriptive characteristics of 102 patients meeting the inclusion criteria

\begin{tabular}{lll}
\hline \multicolumn{1}{c}{ Characteristics } & \multicolumn{1}{c}{ Value } & p Value \\
\hline Median age, yrs (IQR) & $28.5(19.3-48.0)$ & \\
\hline Female (\%) & $53(52.0)$ & \\
\hline Rehemorrhage by AVM location (\%) & & 0.088 \\
\hline Frontal & $1 / 20(5)$ & \\
\hline Parietal & $1 / 22(4.5)$ & \\
\hline Temporal & $0 / 14$ & \\
\hline Occipital & $2 / 4(50)$ & \\
\hline Deep (callosal, basal ganglia, thalamus, & $2 / 20(10)$ & \\
$\quad$ brainstem) & & \\
\hline Periventricular (trigonal) & $0 / 5$ & \\
\hline Cerebellar & $0 / 17$ & \\
\hline Rehemorrhage by AVM eloquence (\%) & & \\
\hline Eloquent & $3 / 51(5.9)$ & \\
\hline Noneloquent & $3 / 51(5.9)$ & \\
\hline Rehemorrhage by AVM drainage & & \\
\hline Deep & $5 / 56(8.9)$ & \\
\hline Superficial & $1 / 45(2.2)$ & \\
\hline Hydrocephalus (\%) & $21(20.6)$ & \\
\hline Smoker (\%) & $31(30.4)$ & \\
\hline Hypertension (\%) & $23(22.5)$ & \\
\hline Values are number of patients $\%)$ & \\
\hline
\end{tabular}

Values are number of patients (\%) unless otherwise indicated.

preference/ lost to follow-up $(n=4)$ and pregnancy $(n=$ $3)$. The treatment paradigm failed in 7 patients $(6.9 \%)$, in $6(5.9 \%)$ of whom failure was due to repeat hemorrhage. The other failure was the result of a decline in neurological status secondary to peri-AVM edema on posthemorrhage Day 20, requiring the patient to undergo surgery within the 4 weeks of the presenting hemorrhage. Half of the repeat hemorrhages occurred within 6 months of the initial hemorrhage, and of these 6 hemorrhagic failures, 5 of them had deep venous drainage. Two patients had repeat hemorrhage on the same day of initial hemorrhage, including 1 patient harboring an associated aneurysm. One patient had repeat hemorrhage 130 days from presentation. Three repeat hemorrhages occurred more than 1 year later in patients whose treatment had been delayed in part due to delayed referral. The majority of repeat hemorrhages were relatively mild, resulting in mild worsening of hemiparesis $(\mathrm{n}=2)$, headache $(\mathrm{n}=1)$, and seizure $(\mathrm{n}$ $=1$ ). The 2 patients who had repeat hemorrhages on the same day of initial hemorrhage had more profound deficits that included decline in Glasgow Coma Scale score requiring intubation and new dense hemiparesis. One of these patients had refractory intracranial pressure and eventually progressed to brain death, and the other patient had a Glasgow Outcome Scale (GOS) score of 3 on last follow-up. No patient suffered rehemorrhage after AVM treatment. The median time from initial hemorrhage to repeat hemorrhage was 248 days (interquartile range [IQR] 33-1364 days). There was a total of 18,740 patient-days observed, yielding a rehemorrhage rate of $0.032 \%$ per day, $0.96 \%$ per month, or $11.5 \%$ per year.
Twelve patients (11.8\%) of the 102 patients had an associated aneurysm. There was 1 patient in this subset who had a repeat hemorrhage. This patient's initial and subsequent hemorrhages were both intraventricular, and it was not clear if the aneurysm or the AVM was the culprit. There were no rehemorrhages in the 1747 patient-days after the aneurysm was secured prior to AVM treatment, while in the 263 patient-days prior to securing of a patient's aneurysm there was $1(8.3 \%)$ hemorrhage out of the 12 patients with associated aneurysms. The rehemorrhage rate for patients with AVM-associated aneurysms in the initial time period was $0.38 \%$ per day or $11.4 \%$ per month.

By removing the group of patients harboring associated aneurysms, we sought to evaluate the risk a patient is exposed to with a ruptured AVM (no associated aneurysm). This evaluation resulted in a rehemorrhage rate of $0.027 \%$ per day, $0.81 \%$ per month, and $9.7 \%$ per year.

A stepwise logistic regression analysis yielded a history of obesity $(p=0.038)$ and the largest single dimension of the AVM $(\mathrm{p}=0.048)$ as predictors of AVM rehemorrhage. Other covariates that were tested but did not appear to be statistically significant predictors included patient sex and age; AVM location, eloquence, and drainage; and history of hypertension, diabetes, cardiac disease, ischemic stroke, hypercholesterolemia, or smoking.

In review of the patient's functional outcome after AVM treatment, $78(90.7 \%)$ of 86 and $60(92.3 \%)$ of 65 patients had favorable GOS score (GOS score $\geq 4)$ at discharge and 6 months from AVM treatment, respectively.

\section{Discussion}

The natural history of a ruptured AVM entails a 6\%$15.8 \%$ risk of rehemorrhage in the 1 st year. ${ }^{1,7,9,23,29}$ This range is what neurosurgeons refer to when determining the management of a patient with a ruptured AVM. Although the rerupture risk diminishes after the 1st year to $1.7 \%-6.2 \%$ per year, cumulatively this risk of rehemorrhage often indicates intervention is warranted to prevent further neurological sequalae. ${ }^{9}$ The present guidelines for AVM treatment suggest that treatment take place in an elective manner but does not specify a protocol. ${ }^{18}$ Our study sought to determine the safety of treating a ruptured AVM beyond the subacute period that was defined as 4 weeks.

Our results paralleled those of prior studies regarding the annual risk of rerupture. In patients with AVMs and no IAs, we found a $9.7 \%$ risk per year of rehemorrhage. Half of our repeat hemorrhages were within the 6-month period after the initial hemorrhage, and this interval, according to some authors, has been regarded as the period representing the highest risk. ${ }^{10}$ The intent of our investigation was to determine the risk a patient was exposed to during the time interval from index hemorrhage to elective treatment, in our institution's treatment paradigm. Our data revealed a $0.027 \%$ per day risk of rehemorrhage in AVM patients not harboring an IA, and this rate increased to $0.032 \%$ per day for the entire 102-patient cohort. This translated to a monthly risk of $0.96 \%$ and $0.81 \%$ for the entire cohort and for those with ruptured AVMs without an IA, respectively.

There were 14 patients excluded from this study be- 
cause they were electively treated with surgery in a subacute manner. These cases were low-grade AVMs with small hemorrhages in neurologically intact patients. The algorithm was abandoned because there was no neurological deficit to rehabilitate and the neurosurgeon of record deemed surgery lower risk. It would not be appropriate to compare these patients to our cohort, as there would be a dramatic selection bias toward simple, minimal-risk surgeries in the acute or subacute surgery group.

There are several studies discussing acute or hyperacute surgery for AVMs. ${ }^{1,2,13,16,19,22,30}$ The time from AVM rupture to surgery ranged from 2 hours to 6 days in these studies. ${ }^{2,13,16,19,22}$ The largest of these included 78 patients categorized into 1 of 3 treatment time intervals: $<24$ hours, 24-48 hours, and $\geq 48$ hours after hemorrhagic presentation. This retrospective study claimed that the patients receiving surgery at $\geq 48$ hours had a worse mean modified Rankin Scale score than did patients in the other 2 time intervals. ${ }^{2}$ However, the study failed to comment on the AVM characteristics of the different groups and the reasons why surgery was delayed $\geq 48$ hours. These data also suggested a trend toward worse outcomes with higher-grade Spetzler-Martin AVMs, and it is possible that the AVMs receiving surgery at $\geq 48$ hours were also the more complicated lesions.

Another study of 27 patients with Spetzler-Martin Grade I-II AVMs treated within 6 days of hemorrhage reported a $7.4 \%$ risk of early rehemorrhage. ${ }^{19}$ This rate was derived from 2 patients in whom rebleeding occurred within 2 hours of their presentation. There was no discussion of whether these patients were included in the 4-patient group that harbored associated aneurysms or if there was a diagnostic study performed to determine the presence of an aneurysm prior to surgery. At this point, there are certainly enough data from studies larger than 27 patients that have determined that the rate of acute rehemorrhage risk of an AVM is not $7.4 \%$.

There is no ambiguity regarding the indication for emergency surgery for a patient who presents in extremis. Life-saving surgery may simply involve a decompressive craniectomy. The clinical challenge is determining which patient should also undergo emergency resection of the AVM. It is our belief that this should be a very small subset of patients requiring aggressive hematoma evacuation that cannot be achieved without avoiding the AVM. It is sometimes difficult to evaluate the effect of early surgery on patient outcomes as the deficits attendant on hemorrhage and surgery will be intertwined at this juncture. Delayed intervention also benefits treatment planning for both radiosurgery and adjunctive embolization. The delay allows for hematoma resorption and better radiological demonstration of the AVM.

Treatment planning, resolution of the hematoma, and rehabilitation of neurological deficits from the initial hemorrhage all play a role in the determination of whether 4 weeks is an appropriate interval of delay prior to intervention. We admit that this is arbitrary but the literature demonstrates that the first 6 months after the initial hemorrhage is the period of highest risk for rehemorrahge. ${ }^{10}$ This is the impetus to intervene early to minimize the risk to which the patient is exposed. The risk of delaying treatment must be weighed against the risk of the intervention. For a patient to incur the additional risk of 5\%-7\% of rehemorrahage while awaiting surgery seems unnecessary. ${ }^{9}$ Furthermore, it is our opinion that there is little surgical benefit in waiting longer than 4 weeks, meaning the surgical environment should have stabilized from the initial hemorrhage by this time. Early surgery may result in residual hematoma that can obscure vision or disorient the neurosurgeon; a brain that often is reactive and friable; and a deficit that may have been rehabilitated but could become permanent. The only way to determine what function a patient may recover is to allow the patient time for recovery prior to surgery. We feel this maximizes the patient's opportunity for favorable neurological outcome.

To balance the risk of rerupture in the first 6 months against the risk of acute or subacute surgery, we propose 4 weeks as a safe period of time for delay. We recognize that this not a novel paradigm for managing AVMs after they present with a rupture, but to our knowledge, there are no data derived from investigating the safety. Further investigation is necessary to determine if a 4-week delay is the optimal time for intervention.

The limitations of the study are its retrospective nature and small cohort of patients, as well as the lack of a control group. The optimal method for investigating the risk of delayed intervention would be to have a multicenter prospective randomized controlled trial with long-term outcomes comparing acute and delayed surgery. The retrospective nature of this study led to a significant portion of patients to be excluded from the cohort based on incomplete data.

We have previously described our treatment paradigm for patients with ruptured brain AVMs and associated IA.? We maintain the opinion that Type IIb and III IAs should be included in the treatment process after an AVM has ruptured. It was our intention to include these patients in this review to quantify any increased risk posed to these patients compared with patients who have AVMs without an identified IA. We found that patients with Type IIb and III IAs and ruptured AVMs had a 14-fold increased risk of rehemorrhage compared to patients with ruptured AVMs without IAs $(0.38 \%$ per day vs $0.027 \%$ per day, respectively). This increased risk exists despite the more aggressive approach of our treatment paradigm when an IA is identified. If these patients were treated in a delayed fashion, this difference might have reached statistical significance. This warrants further investigation and could lead to a paradigm shift with more rapid treatment of those IAs in close proximity to a ruptured cerebral aneurysm without an AVM.

There is a broad range of documented rates of aneurysms associated with brain AVMs in the literature, from $2.7 \%$ to $58 \%$. In our study $11.8 \%$ of patients were found to harbor an aneurysm that we consider an at-risk entity, meaning that the aneurysm is a Type IIb or Type III (distal flow-related or intranidal, respectively). In our previous study, ${ }^{7}$ we documented our experience with these aneurysms and why we regard them as more threatening lesions than their counterparts in unrelated locations (i.e., contralateral hemisphere to the AVM) and proximally located aneurysms (Type I and IIa, respectively). A cohort of only 12 patients with associated aneurysms is a very small number-and this is clearly a limitation to our study-but 
it would be amiss not to include these patients. The literature confirms that these patients are at a higher risk for rehemorrhage, but no guidelines have been set regarding their management. Furthermore, even with the small number of patients as well as our aggressive approach to treating the aneurysm early, we still had a case rehemorrhage before treatment could be implemented. Our goal of management is to eliminate the aneurysm such that the natural history of the lesion is changed to that of a brain AVM without an aneurysm.

We recognize that this retrospective review lacks a cohort for comparison of AVMs treated in an acute or subacute fashion $(<4$ weeks) to validate improved outcomes. However, this has been the paradigm our institution has implemented during the existence of our database and only isolated cases have been managed early. As previously mentioned, these cases were typically small hemorrhages in neurologically intact patients with low-grade AVMs not requiring rehabilitation. It was the decision of the attending surgeon that the risk of early surgery was as low as the risk of our typical protocol leading to early surgery. In these rare instances the benefit was cost efficiency for the patient and hospital.

\section{Conclusions}

We propose that our treatment paradigm using elective intervention for ruptured brain AVMs has a $<1 \%$ added monthly risk to the patient. This is a very low, acceptable amount of added risk. In our opinion, it is unlikely that acute surgery for a ruptured AVM carries such a low risk of morbidity considering factors such as secondary edema and poor visualization of compressed vessels during surgery, both of which could be alleviated with the delay in surgery. Establishing the superiority of acute versus delayed surgery with a high level of certainty would require a prospective, randomized trial with an exceedingly high number of patients. Clearly, our sample size and single-center retrospective analysis limit our study. Multicenter, cooperative studies are imperative for future investigations. In the absence of these large-scale studies, we support delaying treatment for at least 4 weeks to allow the patient to improve neurologically prior to intervention. The subset of patients with a Type IIb or III IA should have the IA treated early if it is possible to do so, and then further management should follow the proposed paradigm. Our data support this as a safe, systematic method of managing AVM patients.

\section{Acknowledgments}

We would like to acknowledge and thank Jerri Thomas and Jamie Thomas, MEd, for their work in maintaining the AVM registry, as well as Suzanne Truex for her illustrations.

\section{References}

1. Aoun SG, Bendok BR, Batjer HH: Acute management of ruptured arteriovenous malformations and dural arteriovenous fistulas. Neurosurg Clin N Am 23:87-103, 2012

2. Bir SC, Maiti TK, Konar S, Nanda A: Overall outcomes following early interventions for intracranial arteriovenous malformations with hematomas. J Clin Neurosci 23:95-100, 2016
3. Choi JH, Mohr JP: Brain arteriovenous malformations in adults. Lancet Neurol 4:299-308, 2005

4. Connolly ES Jr, Rabinstein AA, Carhuapoma JR, Derdeyn CP, Dion J, Higashida RT, et al: Guidelines for the management of aneurysmal subarachnoid hemorrhage: a guideline for healthcare professionals from the American Heart Association/ American Stroke Association. Stroke 43:1711-1737, 2012

5. da Costa L, Wallace MC, Ter Brugge KG, O’Kelly C, Willinsky RA, Tymianski M: The natural history and predictive features of hemorrhage from brain arteriovenous malformations. Stroke 40:100-105, 2009

6. Fleetwood IG, Steinberg GK: Arteriovenous malformations. Lancet 359:863-873, 2002

7. Flores BC, Klinger DR, Rickert KL, Barnett SL, Welch BG, White JA, et al: Management of intracranial aneurysms associated with arteriovenous malformations. Neurosurg Focus 37(3):E11, 2014

8. Friedlander RM: Clinical practice. Arteriovenous malformations of the brain. N Engl J Med 356:2704-2712, 2007

9. Gross BA, Du R: Rate of re-bleeding of arteriovenous malformations in the first year after rupture. J Clin Neurosci 19:1087-1088, 2012

10. Halim AX, Johnston SC, Singh V, McCulloch CE, Bennett JP, Achrol AS, et al: Longitudinal risk of intracranial hemorrhage in patients with arteriovenous malformation of the brain within a defined population. Stroke 35:1697-1702, 2004

11. Inagawa T, Kamiya K, Ogasawara H, Yano T: Rebleeding of ruptured intracranial aneurysms in the acute stage. Surg Neurol 28:93-99, 1987

12. Itoyama Y, Uemura S, Ushio Y, Kuratsu J, Nonaka N, Wada $\mathrm{H}$, et al: Natural course of unoperated intracranial arteriovenous malformations: study of 50 cases. J Neurosurg 71:805809, 1989

13. Jafar JJ, Rezai AR: Acute surgical management of intracranial arteriovenous malformations. Neurosurgery 34:8-13, 1994

14. Kassell NF, Torner JC: Aneurysmal rebleeding: a preliminary report from the Cooperative Aneurysm Study. Neurosurgery 13:479-481, 1983

15. Kassell NF, Torner JC, Jane JA, Haley EC Jr, Adams HP: The International Cooperative Study on the Timing of Aneurysm Surgery. Part 2: Surgical results. J Neurosurg 73:37-47, 1990

16. Kuhmonen J, Piippo A, Väärt K, Karatas A, Ishii K, Winkler $\mathrm{P}$, et al: Early surgery for ruptured cerebral arteriovenous malformations. Acta Neurochir Suppl 94:111-114, 2005

17. Luessenhop AJ, Rosa L: Cerebral arteriovenous malformations. Indications for and results of surgery, and the role of intravascular techniques. J Neurosurg 60:14-22, 1984

18. Ogilvy CS, Stieg PE, Awad I, Brown RD Jr, Kondziolka D, Rosenwasser R, et al: AHA Scientific Statement: Recommendations for the management of intracranial arteriovenous malformations: a statement for healthcare professionals from a special writing group of the Stroke Council, American Stroke Association. Stroke 32:1458-1471, 2001

19. Pavesi G, Rustemi O, Berlucchi S, Frigo AC, Gerunda V, Scienza R: Acute surgical removal of low-grade (SpetzlerMartin I-II) bleeding arteriovenous malformations. Surg Neurol 72:662-667, 2009

20. Pool JL: Timing and techniques in the intracranial surgery of ruptured aneurysms of the anterior communicating artery. $\mathbf{J}$ Neurosurg 19:378-388, 1962

21. Purdy PD, Batjer HH, Samson D: Management of hemorrhagic complications from preoperative embolization of arteriovenous malformations. J Neurosurg 74:205-211, 1991

22. Puzzilli F, Mastronardi L, Ruggeri A, Lunardi P: Early surgical treatment of intracerebral hemorrhages caused by AVM: our experience in 10 cases. Neurosurg Rev 21:87-92, 1998 
23. Rahme R, Weil AG, Bojanowski MW: Early rerupture of cerebral arteriovenous malformations: beware the progressive hemispheric swelling. Med Hypotheses 76:570-573, 2011

24. Samson DS, Hodosh RM, Reid WR, Beyer CW, Clark WK: Risk of intracranial aneurysm surgery in the good grade patient: early versus late operation. Neurosurgery 5:422-426, 1979

25. Stapf C, Mast H, Sciacca RR, Choi JH, Khaw AV, Connolly ES, et al: Predictors of hemorrhage in patients with untreated brain arteriovenous malformation. Neurology 66:1350-1355, 2006

26. Stapf C, Mohr JP, Pile-Spellman J, Solomon RA, Sacco RL, Connolly ES Jr: Epidemiology and natural history of arteriovenous malformations. Neurosurg Focus 11(5):e1, 2001

27. Steudel WI, Lorenz R, Berkefeld J: Acute operation on arteriovenous malformation with hematomas-report on six cases. Neurochirurgia (Stuttg) 35:26-30, 1992

28. Troupp H, Marttila I, Halonen V: Arteriovenous malformations of the brain. Prognosis without operation. Acta Neurochir (Wien) 22:125-128, 1970

29. Yamada S, Takagi Y, Nozaki K, Kikuta K, Hashimoto N: Risk factors for subsequent hemorrhage in patients with cerebral arteriovenous malformations. J Neurosurg 107:965972, 2007

30. Zacharia BE, Vaughan KA, Jacoby A, Hickman ZL, Bodmer D, Connolly ES Jr: Management of ruptured brain arteriovenous malformations. Curr Atheroscler Rep 14:335-342, 2012

\section{Disclosures}

Dr. Welch is a consultant for Stryker Neurovascular. He receives royalties from Peter Lazic, Inc., for designing an aneurysm clip, and he is a proctor for Covidien for the Pipeline flow-diversion device.

\section{Author Contributions}

Conception and design: Beecher, Ban, Welch. Acquisition of data: Beecher, Lyon, Ban, McDougall, Whitworth, White, Samson, Batjer, Welch. Analysis and interpretation of data: Beecher, Lyon, Ban, Vance, Welch. Drafting the article: Beecher, Lyon, Ban, Vance, McDougall, Welch. Critically revising the article: all authors. Reviewed submitted version of manuscript: Beecher, Ban, Vance, McDougall, Batjer, Welch. Approved the final version of the manuscript on behalf of all authors: Beecher. Statistical analysis: Lyon, Ban. Administrative/technical/material support: Beecher, Whitworth, White, Samson, Batjer, Welch. Study supervision: Beecher, Welch.

\section{Supplemental information}

\section{Previous Presentations}

Presented in abstract and oral form at the 2016 CNS Annual Meeting, September 24-28, 2016, San Diego, California.

\section{Correspondence}

Jeffrey S. Beecher, 351 Ashland Ave. Upper, Buffalo, NY 14222. email: drj.beecher@gmail.com. 